\title{
RECHERCHES EXPÉRIMENTALES SUR QUELQUES PRODUITS EMPLOYÉS DANS LE TRAITEMENT DE LA DYSENTERIE AMIBIENNE
}

\author{
Par J. SAUTET
}

Avant de partir en mission pour l'Amérique du Sud, le prof. Brumpt nous avait demandé de comparer l'action de l'émétine sur l'Entamæba dysenteriæ et l'Entamæba dispar en culture.

Personne n'ayant encore étudié in vitro l'action de l'émétine sur $l^{\prime} E$. dispar, notre maître pensa qu'il était peut-être possible de donner, par cette méthode, un caractère différentiel nouveau à cette dernière espèce, nettement différenciée par ailleurs.

Entraîné par notre sujet nous avons, en outre, étudié l'action de différents médicaments sur les cultures d'amibes et ètabli une discussion à propos de notre expérimentation.

Le traitement de Ia dysenterie amibienne a fait ces dernières années des progrès considérables : aussi les cas de guérison rapportés par de nombreuses statistiques sont-ils impressionnants. Mais on a peut-être abusé du terme de "médicament spécifique " et on en est convaincu quand on regarde la grande quantité de produits préconisés.

Cependant, pour cette question du traitement, l'avis des médecins doit être le premier considéré.

Que nous disent les chiffres qu'ils publient ? C'est qu'en dehors des multiples produits employés seulement dans des cas particuliers (quinine, acriflavine, novarsénobenzol, tréparsol, auremetine, nitrate d'argent, etc.), on possède actuellement pour combattre l'amibe trois médicaments puissants, qui sont : dans les cas aigus, l'émétine, et dans les cas chroniques, le stovarsol et le yatren 105.

Or, parmi ces 3 médicaments, dont l'activité est certaine, il en est deux qui le semblent peu dans les expériences sur les amibes en culture. Aussi devons-nous toujours nous rappeler que même si des résultats expérimentaux ne semblent pas entièrement confirmer la valeur d'un médicament, nous sommes obligés de reconnaître ses propriétés curatives, lorsqu'il permet au médecin d'enregistrer des guérisons irréfutables.

Annales de Parasitologie, T. V, $\mathrm{N}^{\circ} 4$. $^{\prime}-1^{\text {er }}$ octobre 1927 , p. 329-343. 
Le rôle de l'expérimentateur se borne donc à chercher comment les médicaments agissent sur le parasite, que cette action soit directe ou indirecte.

Des recherches anciennes nous ne retiendrons que celles de Vedder et de Rogers ; et, plus près de nous, celles de Sellard et Leiva. Malheureusement, comme à cette époque la culture des amibes était inconnue (tout au moins celle de l'Entamoba dysenteriæ), la recherche du pouvoir " amibicide " des diverses substances était difficile à constater sur des organismes si fragiles.

Mais après la découverte de Boeck et de Drbohlav, cette lacune fut comblée et plusieurs travaux importants parurent sur l'action de diverses substances sur les cultures d'amibes. Ce furent d'abord celui de Kofoid et Wagener en 1925, puis de Dobell et Laidlaw en 1926, et de Vogel en 1927. Mais on est surpris de trouver de grands écarts dans les conclusions de ces différents auteurs et c'est alors que l'on s'aperçoit du peu de valeur. de cette méthode dont on attendait beaucoup.

Nous avons repris quelques-unes de ces expériences, et cela d'aus tant plus facilement que des chiffres approximatifs nous étaient fournis par les travaux précédents, nous évitant ainsi bien des tâtonnements.

Comme nous nous y attendions, les résultats obtenus ont souvent été discordants : aussi tout notre effort s'est-il porté vers la recherche des causes amenant de telles variations dans les chiffres.

\section{Expérimentation de diverses substances sur les cultures d'amibes}

1. Techniques. - Les recherches suivantes ont été faites sur des amibes ( $\boldsymbol{E}$. dysenteriæ et $\boldsymbol{E}$. dispar) cultivées suivant la méthode de Boeck et Drbohlav, sur milieux de Dorset ou L.-E.-S. (Locke-EggSérum), additionnés d'amidon de riz en grains. Aux tubes, contenant la partie solide du milieu, nous ajoutons le milieu liquide dans lequel se trouve en solution le médicament à étudier (1), en proportions voulues. Le tout est porté à l'étuve à $37^{\circ}$ : on laisse pendant 1 ou 2 heures, puis on ensemence, en même temps qu'un tube témoin. Les cultures sont alors examinées le lendemain.

Les chiffres d'amibes, que nous donnons comme résultats, sont obtenus en examinant entre lame et lamelle une goutte provenant du râclage de la surface solide du milieu de culture : examen fait

(1) Les produits employés proviennent des maisons suivantes :

Emétine, céphéline, ipéca : Laboratoires Clin, Paris. Stovarsol, 914, 606, iodure double de bismuth et d'émétine : Etablissement Poulenc, Paris. Yatren 105 : Institut Behring, Marbury-Lahn. 
à l'étuve chauffante à l'objectif 2 et à l'oculaire 9 d'un niicroscope Stiassnie et en comptant le nombre d'amibes dans un seul champ.

Renseignements sur les souches d'amibes employées. - Pour nos expériences, nous avons employé 3 souches dont voici l'origine exacte :

I. Culture $1(S$.$) . - Les amibes de cette culture proviennent des selles$ glaireuses et sanglantes d'un malade C. atteint d'une dysenterie amibienne contractée il y a 1 an 1/2 en Indochine et rebelle jusqu'en mai 1927 à la thérapeutique habituelle, date à laquelle une série d'émétine amena une stérilisation.

II. Culture $2(S)$. - Culture d'amibes dysenteriques à partir d'un malade $P$. atteint depuis 2 ans d'une dysenterie amibienne aiguë, rebelle à toute thérapeutique, dysenterie contractée au Maroc.

III. Culture 3 (S.). - Les amibes de cette culture proviennent de kystes à 4 noyaux chez un porteur sain. Ces kystes, isolés et ensemencés suivant la méthode de «Yorke et Adams », nous ont été procurés par notre ami èt collègue Bidegaray, avec qui nous avons étudié ce cas. "Observation « du malade A. porteur de ces kystes : Age : 20 ans. Né à Lille. Actuelle« ment ce malade est hospitalisé au Val-de-Grâce, dans le service du \& $\mathrm{P}^{\mathrm{r}}$ Jausion, pour blennorragie. Ce jeune homme n'a jamais quitté la \& France et n'a eu aucun contact avec des coloniaux. Il ne présente aucun « passé intestinal, sauf une constipation assez opiniâtre 》 (Bidegaray). A partir de ce malade nous faisons des cultures sur L. E. S. avec amidon de riz. Puis nous avons inoculé plusieurs fois consécutives ces cultures au chat.

$1^{\circ}$ Le chat $\mathrm{n}^{\circ} 1$ B. S., pesant $600 \mathrm{gr}$., est inoculé, suivant la méthode de Drbohlav, le 20 janvier 1927 , avec 6 tubes de cultures aux $5^{\circ}$ et $6^{\circ}$ passages, riches de 5 à 50 amibes. Le 21, le chat est débouché, il ne présente pas d'amibes, aussi nous le réinoculons le 25 janvier avec 6 autres tubes riches de 20 à 50 amibes. Ce chat meurt le 30 janvier sans avoir présenté la moindre amibe dans ses selles. L'autopsie montre un intestin parfaitement normal.

$2^{\circ}$ Le chat $\mathrm{n}^{\circ} 2$ B. S., pesant $650 \mathrm{gr}$., est inoculé suivant la même méthode le 2 février 1927 avec 6 autres tubes au $16^{\circ}$ passage et contenant un grand nombre d'amibes. Ce chat reste encore complètement négatif. Aussi nous le réinoculons le 7 février avec 8 tubes de cultures au $23^{\circ}$ passage. Les selles continuent à se montrer négatives. Enfin nous faisons une nouvelle inoculation le 19 février avec 8 tubes de cultures au $33^{\circ}$ passage. Le chat débouché le 20 est négatif. Le 21, nous voyons 4 amibes très mobiles et non hématophages dans les selles. Le 22 et les jours suivants, l'examen quotidien se montre toujours négatif.

Ce chat est alors sacrifié le 9 mars et l'autopsie montre un intestin normal.

$3^{\circ}$ Le chat $n^{\circ} 3$ B. S., pesant 400 gr., est alors inoculé le 14 mars avec 4 tubes de cultures au $58^{\circ}$ passage contenant 10 à 100 amibes. Il est débouché le 16 mars et ne présente aucune amibe. Il meurt spontanément 
le 22 mars, toujours négatif et l'autopsie ne montre aucune lésion intestinale.

Malgré le passage sur milieux de culture avec amidon, ce cas est bien pour le $\mathrm{P}^{\mathrm{r}}$ Brumpt un cas d'E. dispar, vu le nombre, la répétition des inoculations au jeune chat èt le pouvoir pathogène si faible, puisque dans un seul examen quelques amibes ont seulement été constatées. Pour des raisons indépendantes de nous, les selles du malade n'ont pu être inoculées directement au chat. Aussi le $\mathrm{P}^{\mathrm{r}}$ Brumpt pense-t-il que si cette inoculation avait été faite, on aurait logiquement pu avoir des infections passagères très nettes comme celles qu'il a décrites dans le Bulletin de l'Académie de Médecine en 1925 (XCIV, $\mathrm{n}^{\circ} 35$ ) et par là-même confirmer ce cas d'Entamœba dispar.

2. Action de l'ipéca. - Pour connaître l'action de Y'ipéca nous avons dû ajouter directement une quantité de poudre déterminée dans chaque tube, avant l'ensemencement, ce qui nous a permis, dans ce cas, de n'avoir que des résultats approximatifs.

L'examen des cultures a été fait le lendemain de notre repiquage, comme de coutume.

Des tubes témoins ensemencés en même temps et en partant de la même souche nous ont permis de comparer les résultats.

Le tableau résume ce que nous avons fait :

\begin{tabular}{|l|l|l|}
\hline Concentration de l'ipéca & $\begin{array}{c}\text { Examen de la culture } \\
\text { 24 heures aprés }\end{array}$ & Examen de tube témoin \\
\hline & 0 & $10-20$ \\
1 p. 50 & 1 en tout par lamelle & $10-20$ \\
1 p. 100 & 1 & 20 \\
1 p. 200 & $10-20$ & 20 \\
1 p. 500 & & \\
\hline
\end{tabular}

L'ipéca semble done n'avoir qu'une influence faible sur les ámibes, sauf à de très grandes concentrations : 1 p. 50, 1 p. 100.

3. Action de l'émétine. - Contrairement à certains auteurs nous avons trouvé à l'émétine une toxicité, pour les amibes, plus faible que celle généralement admise.

Mais nous savons trop la difficulté d'obtenir des expériences comparables avec des cultures aussi souillées et variableš, pour nous étonner de résultats discordants :

Nous avons surtout étudié l'action comparée de l'émétine sur l'E. dysenteriæ et l'E. dispar. Voici nos résultats pour l'E. dispar (Culture 3 S.) : 


\begin{tabular}{|c|c|c|}
\hline CONCENTRATION DE L'ÉMÉTINe & $\begin{array}{l}\text { EXaMEN DB LA CULTCRE } \\
24 \text { HEURES APRÈS }\end{array}$ & EXAMEN DU TUBE TÉMOIN \\
\hline $\begin{array}{rrr}1 & \text { p. } & 500 \\
1 & \text { p. } & 1.000 \\
1 & \text { p. } & 10.000\end{array}$ & $\begin{array}{l}0 \\
0-1 \\
-1-5\end{array}$ & $\begin{array}{r}210 \\
10-15 \\
10-15\end{array}$ \\
\hline
\end{tabular}

Voici, d'autre part, nos chiffres pour l'E. dysenteriæ (Culture 1) :

\begin{tabular}{|c|c|c|}
\hline CONCENTRATION DE L'ÉMÉTINE & $\begin{array}{l}\text { EXAMEN DE LA CULTURE } \\
24 \text { HEURES APRÈS }\end{array}$ & EXАMEN dU TUBE TÉMOIN \\
\hline 1 p. 1.000 & $0-1$ & $\begin{array}{r}1-20 \\
15-90\end{array}$ \\
\hline 1 p. 10.000 & $5-6$ & \\
\hline
\end{tabular}

L'E. dysenterix et l'E. dispar semblent donc se comporter d'une façon toute semblable vis-à-vis de l'émétine in vitro et il est impossible de trouver là un caractère différentiel entre les 2 espèces par cette méthode.

4. Action de la céphéline. - Avec les souches d'amibes prẻcédentes nous avons, par contre, trouvé les mêmes résultats que Dobell. Comme lui, disons que la céphéline est plus nocive que l'émétine pour les amibes en culture.

\begin{tabular}{|c|c|c|}
\hline $\begin{array}{l}\text { CONCENTRATION } \\
\text { EN CÉPHÉLINE }\end{array}$ & $\begin{array}{l}\text { EXAMEN DE LA CULTURE } \\
24 \text { HEURES APRÈs }\end{array}$ & EXAMEN DU TUBE TÉMOIN \\
\hline 1 p. 25.000 & 0 & 50 \\
\hline 1 p. 50.000 & 0 & 100 \\
\hline 1 p. 75.000 & $0-2$ dans toute une lamelle & 50 \\
\hline 1 p. 100.000 & 20 & 50 \\
\hline 1 p. 200.000 & $30-40$ & 50 \\
\hline
\end{tabular}

5. Action de l'iodure double d'émétine et de bismuth. - L'iodure double d'émétine et de bismuth se montre assez peu actif pour la destruction des amibes en culture. 
On trouve:

\begin{tabular}{|c|c|c|}
\hline $\begin{array}{l}\text { CONCENTRATION EN TODURE } \\
\text { DOUBLE }\end{array}$ & $\begin{array}{l}\text { EXAMEN DE LA CULTURE } \\
24 \text { HEURES APRÉs }\end{array}$ & EXAMEN DU TUBE TÉMOIN \\
\hline 1 p. 50.000 & $5-6$ & 10 \\
\hline 1 p. 1.000 & $1-2$ & $3-10$ \\
\hline 1 p. 500 & $0-1-2-0$ & $10-20$ \\
\hline 1 p. 100 & 0 & $10-20$ \\
\hline
\end{tabular}

Mais comment agit ce produit ? car le lendemain de l'ensemencement la culture devient noire par dépôt probable de bismuth ; il est, en effet, possible qu'il se passe dans les tubes de culture ce qui se produit chez l'homme, c'est-à-dire un dédoublement du corps avec mise en liberté d'émétine et, dépôt de bismuth.

Ces résultats ne sont done plus pour nous surprendre car ils concordent assez bien avec ceux obtenus pour l'émétine.

6. Action du stovarsol. - A côté de la réelle efficacité du stovarsol dans l'amibose : nous ne lui reconnaissons qu'une faible toxicité envers les cultures d'amibes.

Voici les résultats obtenus avec ce produit :

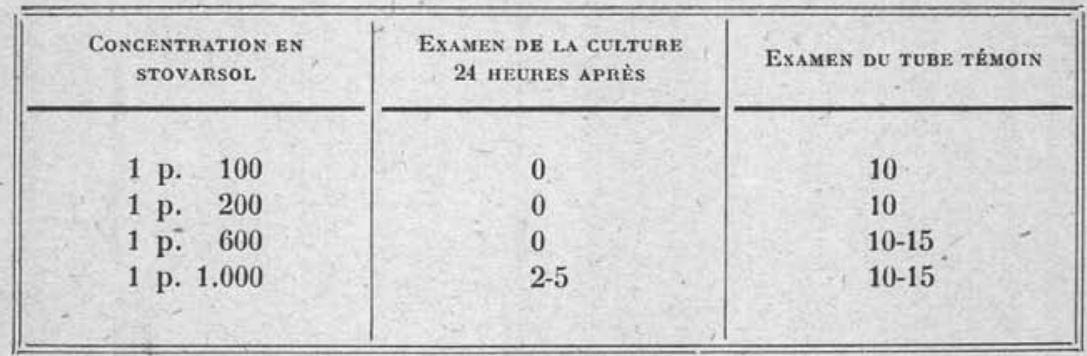

Le stovarsol n'agit donc pour empêcher la culture des amibes qu'à une concentration supérieure à 1 p. 1.000 : dose jamais atteinte au cours d'un traitement anti-dysentérique.

Doit-on en conclure que ce produit est un mauvais médicament contre les amibes? Non, évidemment, car les chiffres ne valent que pour les cultures, c'est-à-dire ne valent pas grand'chose, comme nous le démontrerons plus loin.

7. Action du novarsénobenzol. - Le novarsénobenzol nous a.semblé être très actif sur les amibes.

Est-ce par action directe ou indirecte après décomposition en 
présence des multiples bactéries du milieu de culture ? Il nous est encore impossible de répondre.

\begin{tabular}{|c|c|c|}
\hline $\begin{array}{l}\text { ConcENTRATION EN } \\
\text { NOVARSÉ OBB EZOL. }\end{array}$ & $\begin{array}{l}\text { EXAMEN DE LA CELTURE } \\
24 \text { HEURES APRÉS }\end{array}$ & EXAMEN DU TUBE TÉMOIN \\
\hline 1 p. $\quad 100$ & 0 & $5-10$ \\
\hline 1 p. 500 & 0 & 5 \\
\hline 1 p. $\quad 1.000$ & 0 & $5-20$ \\
\hline 1 p. $\quad 5.000$ & 0 & $5-20$ \\
\hline 1 p. 10.000 & 0 & $2-15$ \\
\hline 1 p. 50.000 & $1-2$ & $2-30$ \\
\hline 1 p. 100.000 & $2-10$ & $10-30$ \\
\hline
\end{tabular}

8. Action du yatren 105. - A côté du stovarsol, il nous paraît intéressant d'étudier le yatren 105 , employé comme lui surtout au cours des dysenteries chroniques, rebelles à l'action de l'émétine.

Là encore, nous avons dû reconnaître que le pouvoir anti-amibien in vitro diffère beaucoup du pouvoir in vivo et ne peut, dans la pratique, donner aucune indication, si on se borne à ces simples constatations.

Nos résultats sont les suivants :

\begin{tabular}{|c|c|c|}
\hline Concentration en yatren & $\begin{array}{l}\text { ExameN dÈ LA CULTURE } \\
24 \text { HEURES APRÈs }\end{array}$ & EXAMEN DU TUBE TÉMOIN \\
\hline 1 p. 100 & 0 & 20 \\
\hline 1 p. 200 & 0 & $10-15$ \\
\hline 1 p. 400 & 4 dans toute une lamelle & 20 \\
\hline 1 p. 600 & $1-6-10$ & $5-20$ \\
\hline
\end{tabular}

9. Action du sublimé. - A côté de l'action des substances réputées anti-amibiennes par les médecins, nous avons expérimenté certains corps antiseptiques courants :

\begin{tabular}{|c|c|c|}
\hline CONCENTRATION EN SUBLIMÉ & $\begin{array}{c}\text { EXaMEN DE LA CULTERE } \\
24 \text { HEURES APRÈs }\end{array}$ & EXAMEN dU TUBE TÉmoIN \\
\hline $1 \mathrm{p}$ & 0 & 50 \\
\hline $1 \mathrm{p}$. & 10 & 10 \\
\hline 1 p. $\quad 1.000$ & $2-3$ & 20 \\
\hline 1 p. 2.000 & 5 & 50 \\
\hline 1 p. 200.000 & 10 & $10-20$ \\
\hline
\end{tabular}


Le sublimé ne semble pas avoir une action très intense șur les amibes, malgré son pouvoir toxique habituel très élevé.

10. Action de l'iode. - L'iode qui se fixe si facilement sur les amibes, aussi bien à l'état de kystes que de formes végétatives, nous a' semblé intéressant à étudier ?

Mais l'iode étant insoluble dans la solution "locke-sérum ", pour le dissoudre, nous avons été obligés d'ajouter de l'iodure de potassium. Ce produit (aux doses employées) ne semble pas nuire beaucoup au développement des amibes. Du reste si l'on considère l'iode comme " médicament théorique possible ", on est bien obligé d'admettre son association à un solvant pour son administration facile et il nous semble rationnel d'étudier le mélange (I et KI).

Voici les résultats trouvés :

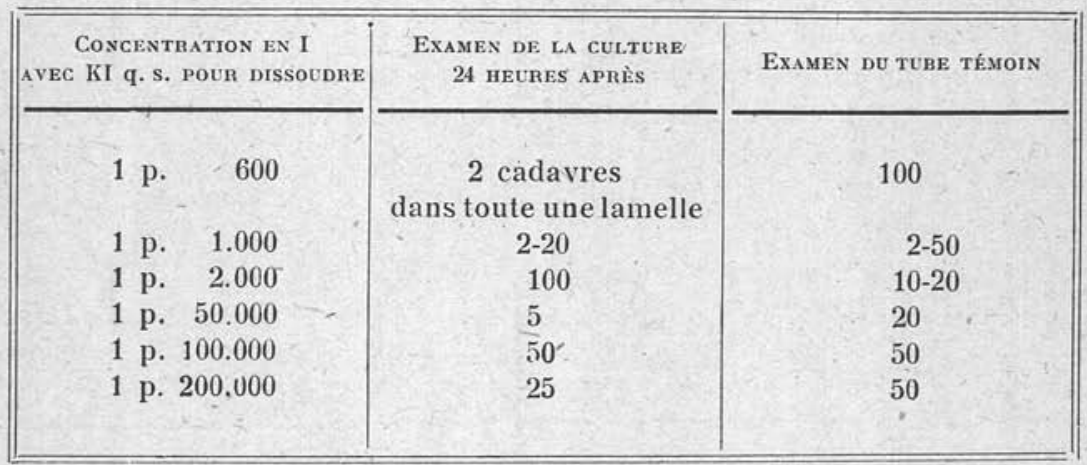

L'amibe nous paraît relativement peu sensible à l'action de l'iode avec l'iodure de potassium, ce qui peut étonner à priori. Or nous allons voir que ces résultats ne sont en réalité pas justes et demandent certaines rectifications.

\section{Discussion des résultats obtenus}

1. Remarques générales sur la valeur de la méthode employée dans ce travail. - Ces remarques aussi vieilles que la méthode, nous semblent devoir être rappelées.

Nous commencerons cette discussion en essayant d'élucider la valeur réelle de l'iode comme amibicide (ou tout au moins d'une façon approximative). Nous avons du reste étudié ce corps'dans le but de montrer les causes d'erreur possibles, qui, dans ce cas particulier, sont faciles à mettre en évidence. Ce qui nous permettra de dire : puisque ce produit subit des transformations en présence 
des milieux de culture, n'en est-il pas de même pour les autres ? Il s'en suivra done une grande prudence dans les conclusions que nous tirerons de l'étude de l'action des médicaments sur les cultures : tel est le but principal de notre discussion et nous n'ambitionnons pas une détermination exacte du pouvoir amibicide des diverses substances, que seul un chimiste exercé serait dans la possibilité d'entreprendre?

2. Que doit-on penser de l'action anti-amibienne de l'iode ? Dans le cas de l'iode les causes d'erreur sautent aux yeux, à cause des réactions colorantes qu'entraine son adjonction aux milieux. Les tubes de Dorset et L. E. S. de Boeck et Drbohlav communément employés sont constitués en majeure partie par de l'albumine : aussi est-on certain d'avance d'avoir des combinaisons chimiques avec l'iode, comme le dit le prof. Carnot dans son Précis de Thérapeutique: "...la plupart des antiseptiques, étant des coagulants " de l'albumine, agissent mal en milieu albumineux. Par exemple " si l'on compare le pouvoir antiseptique du sublimé, du formol, " sur des cultures en bouillon et en sérum, les chiffres sont très " différents : très antiseptiques en bouillon, ces corps ont un pou" voir antiseptique réduit en milieu albumineux. Il y a là un point " de première importance... "

Dans nos expériences nous sommes partis d'abord d'une solution d'iode à 1 p. 1.000 dans un liquide ainsi constitué :

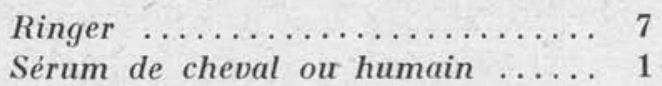

avec une quantité d'iodure de potassium nécessaire pour dissoudre.

Cette solution est ajoutée à la partie solide d'un tube de Dorset : après un jour de contact le liquide est beaucoup moins jaune ; de toute évidence il s'est transformé.

$\mathrm{Si}$, à ce moment on retire le liquide et qu'on l'agite dans un tube à essai avec du chloroforme : au lieu de voir ce dernier prendre une teinte mauve, il reste parfaitement clair: l'iode n'existe plus à l'état libre.

Comme nous n'ensemençons les amibes dans les tubes qu'après les avoir laissés pendant une heure à l'étuve nous avons cherché au contact de combien d'iode libre se trouvaient alors les amibes :

$1^{\circ}$ Nous prenons d'abord une solution approximative d'iode dans le Ringer-sérum.

$2^{\circ}$ Nous dosons cette solution par l'hyposulfite de soude en présence d'empois d'amidon comme indicateur coloré : 
Nous trouvons comme résultat:

solution d'iode à : $\quad \frac{0,6858}{600}$.

A cette concentration, entre lame et lamelle, les amibes meurent assez rapidement.

$3^{\circ}$ On recouvre de cette solution le milieu solide (œuf coagulé) pendant une heure.

$4^{\circ}$ On dose à nouveau la quantité d'iode en liberté dans ce liquide.

On trouve alors :

solution d'iode à :

$$
\frac{0,381}{600} \text {. }
$$

Les amibes meurent dans ce tube, on peut donc dire qu'elles sont beaucoup plus sensibles à l'action de cet antiseptique que les chiffres d'iode ajoutés ne le laissaient entendre.

Car au lieu d'une mort à : $\frac{0,68}{600}$,

On a une mort à : $\quad \frac{0,38}{600}$.

Et peut-être moins encore car avec le temps la concentration en iode libre dans les tubes diminue pour finalement arriver à 0 .

3. Le stovarsol a-t-il une action directe sur les amibes ? - Dans sa thèse (Paris, 1925), Sanglier disait : "Il ne semble pas irrationnel de rattacher le mode d'action encore méconnu du stovarsol à un processus sensiblement analogue à celui que subit le novarsénobenzol " (formation d'un complexe biologique selon l'hypothèse de Levaditi).

Ces idées étant les nôtres nous avons essayé de les expliquer par l'expérience.

Nous avons en partie suivi la méthode de Levaditi.

Technique employée. - $1^{\circ}$ On sacrifie un chat de 600 à 700 grammes par saignée (chats 1 bis, 2 bis et 3 bis $\mathrm{S}$.). On recueille le foie (sans sa vésicule biliaire), l'estomac et l'intestin grèle, dans des récipients différents.

$2^{\circ}$ On hache et on triture dans un mortier, séparément, ces trois organes auxquels on a ajouté 20 centimètres cubes de liquide de Ringer.

On centrifuge rapidement ces trois solutions pour enlever les grosses particules.

On recueille dans 3 tubes les solutions surnageant.

$3^{\circ}$ On répartit ces solutions comme il suit :

a) émulsion de foie, 2 cc. ; 
solution de stovarsol à 1 p. X, dans Ringer-sérum, 2 cc. ;

b) émulsion de foie, 2 cc. ;

solution de Ringer-sérum, 2 cc. ;

c) émulsion d'estomac 2 ce.;

solution de stovarsol à 1 p. X, dans Ringer-sérum, 2 cc. ;

d) émulsion d'estomac, 2 cc. ;

solution de Ringer-sérum, 2 cc. ;

e) émulsion d'intestin grêle, 2 cc. ;

solution de stovarsol à II p. X, dans Ringer-sérum, 2 cc. ;

f) émulsion d'intestin grêle, 2 cc. ;

solution de Ringer-sérum, 2 cc. ;

A chacun de ces tubes (laissés pendant 1 heure à $37^{\circ}$ ) on ajoute 7 gouttes de culture d'amibe riche, en même temps qu'on ense. mence des tubes témoins.

Le taux des solutions de stovarsol employées est indiqué pour chaque expérience. Puis les cultures sont examinées le lendemain. Voici le résultat de nos expériences sur l'E. dysenteriæ (culture $1 \mathrm{~S}$.$) :$

\begin{tabular}{|c|c|c|c|c|}
\hline \multirow{2}{*}{$\begin{array}{l}\text { NATURE DU TUBE } \\
\text { DE CULTURE }\end{array}$} & \multicolumn{4}{|c|}{$\begin{array}{c}\text { EXAMEN } 24 \text { HEURBS APRÈS L'ENSEMENCEMENT } \\
\text { STOVARSOL à : }\end{array}$} \\
\hline & 1 p. 2.000 & 1 p. 4.000 & 1 p. 2.000 & 1 p. 4.000 \\
\hline L.-E.- $\quad$ et estomac .. & $15-30$ & $\ldots \ldots \ldots$ & 10 & 1 \\
\hline Estomac et stovarsol & $5-10$ & . & $?$ & \\
\hline Tube témoin ........ & $15-100$ & 0 & $1-7$ & \\
\hline Intestin grêle L.-E.-S. & $?$ & $\cdots$ & $\frac{1}{50}$ & \\
\hline $\begin{array}{l}\text { Intestin grêle et sto- } \\
\quad \text { varsol } \ldots \ldots \ldots \ldots \ldots\end{array}$ & $?$ & & $?$ & \\
\hline Tube témoin ... & $2-5$ & $\ldots$ & & \\
\hline Foie L. E. S... & $2-3$ & $2-3$ & $2-20$ & $5-20$ \\
\hline Foie et stovarsol & $\begin{array}{c}2 \text { en tout } \\
\text { par lamelle }\end{array}$ & 0 & $\begin{array}{c}3 \text { en tout } \\
\text { par lamelle }\end{array}$ & $\begin{array}{l}7 \text { en tout. } \\
\text { Aspect picqueté. } 2 \\
\text { seulement sont } \\
\text { vivantes d'une } \\
\text { façon certaine. }\end{array}$ \\
\hline Tube témoin. & $10-15$ & & & \\
\hline
\end{tabular}


Quelles conclusions allons-nous tirer de ces expériences? Les chiffres ne sont pas assez formels pour permettre d'être affirmatif. Cependant il nous semble possible d'admettre une plus grande toxicité du stovarsol en présence du foie, que normalement.

Nous devons aussi remarquer sur ce tableau, le peu d'influence du milieu stomacal sur les amibes mobiles ; et l'on peut se demander si l'infection par la voie buccale, en partant de formes végétatives, n'est pas possible, contrairement à l'opinion de la plupart des auteurs.

Quant à l'action de l'extrait d'intestin grêle sur les cultures, son action défavorable en l'absence de toute substance toxique nous demandera de nouvelles expériences.

4. Ghlorhydrate d'émétine et sérum. - Les doses diverses d'émétine ne tuent les amibes qu'après un contact prolongé avec les cultures.

Ceci semble à première vue en désaccord avec les expériences de Vedder et Rogers, car a priori on ne s'attend pas à cette action assez lente de l'émétine.

D'autre part, nous nous trouvons en clinique en présence de faits, qui sont, là aussi, différents. Les médecins sont en effet presque unanimes à constater l'action très rapide de doses même relativement très faibles de chlorhydrate d'émétine, par rapport au poids du sujet traité.

Comment expliquer cette apparente contradiction ?

Nous avons dans ce but émis l'hypothèse suivante : le sang contenant du chlorhydrate d'émétine n'est-il pas plus toxique qu'une solution aqueuse de ce dernier produit ?

-Voici les quelques expériences que nous avons ébauchées dans ce but :

On injecte par voie intra-veineuse (veine fémorale) à un chat de 700 grammes (chat $\mathrm{n}^{\circ} 5$ bis S.) une solution de chlorhydrate d'émétine ( $0 \mathrm{gr}$. 10 dans $2 \mathrm{~cm}^{\mathrm{s}-}$ d'eau physiologique). On pousse lentement l'injection. Le chat meurt avec une vaso-constriction intense. On recueille rapidement le sang dont on étudie la toxicité sur des cultures d'amibes dans des tubes de L.-E.-S. à $37^{\circ}$ pendant 24 heures.

On ajoute quelques gouttes (leur nombre est donné dans le tableau), soit de sanng total, soit de sérum, soit de globules. L'examen des tubes est fait le lendemain en même temps que celui de tubes témoins, qui permettent d'interpréter les résultats. 
Tableau des résultats obtenus :

\begin{tabular}{|c|c|c|c|}
\hline $\begin{array}{l}\text { PaRtie de SANG AJOUtÉE } \\
\text { OU SÉnUM }\end{array}$ & $\begin{array}{c}\text { NoMBre dE } \\
\text { GOUTtES AJOUTÉES }\end{array}$ & $\begin{array}{l}\text { EXAMEN APRÈs } \\
24 \text { nEURES }\end{array}$ & $\begin{array}{l}\text { EXAMEN DU TUBE } \\
\text { TÉMOIN }\end{array}$ \\
\hline Sang normal............ & VII & $5-10$ & $5-10$ \\
\hline $\begin{array}{l}\text { Sang du chat } 5 \text { bis } \mathrm{S} . \\
\text { (Emétine) } \ldots \ldots \ldots \ldots \ldots\end{array}$ & $\mathrm{V}$ & $\begin{array}{l}3 \text { kystes et qq. } \\
\text { formes mobiles }\end{array}$ & 5 \\
\hline $\begin{array}{r}\text { Globules du chat } 5 \text { bis } \mathrm{S} \text {. } \\
\text { (Eméline) } \ldots \ldots \ldots \ldots \ldots\end{array}$ & V & $1-4$ & 10 \\
\hline Sérum normal d'un chat. & $\mathrm{XX}$ & $1-2$ & 5 \\
\hline $\begin{array}{r}\text { Sérum du chat } 5 \text { bis } \mathrm{S} \text {. } \\
\text { (Emétine)............. }\end{array}$ & VII & 0 & $5-20$ \\
\hline id. & V & 0 & $5-15$ \\
\hline id. & III & 0 & $2-10$ \\
\hline id. & II & $\begin{array}{l}3 \text { dans toute } \\
\text { unelamelle }\end{array}$ & $1-10$ \\
\hline id. & I & $\begin{array}{l}4 \text { dans toute } \\
\text { unelamelle }\end{array}$ & $10-20$ \\
\hline
\end{tabular}

Or, un tube contient environ 1 gramme de liquide " Ringer sérum ", de plus, chaque goutte ajoutée avec notre pipette pèse (sérum du chat) : 0 gr. 05 . Enfin on peut admettre que les $0 \mathrm{gr} .10$ d'émétine injectée se sont répartis dans le sang (et ne se sont guère encore fixées sur les organes), c'est-à-dire dans environ $70 \mathrm{gr}$. Ces chiffres sont très approximatifs, mais il est buien certain que la quantité d'émétine, qui serait arrivée au contact des lésions intestinales, aurait été encore beaucoup plus faible que celle que nous donnons.

On peut donc dire après avoir fait le calcul dans chaque cas que les amibes en culture sont rapidement tuées en 24 heures par l'émétine à I pour X, en solution dans le sérum.

VII gouttes, sérum et émétine ...... 0 amibes......... 4,9 p. 10.000

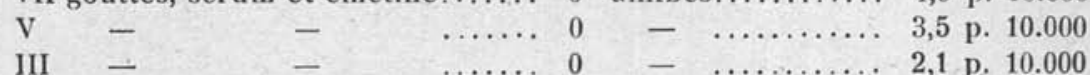


II goultes, sérum et émétine ...... 3 en tout.......... 1,4 p. 10.000

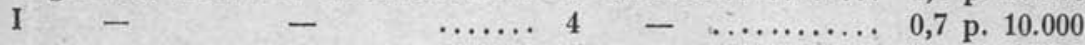

Donc à des concentrations égales dans des solutions différentes, l'action de l'émétine est plus intense et rapide quand elle se trouve dans le sérum et de plus cette action est beaucoup plus rapide.

De plus comme nous nous y attendions, d'après les travaux de Maurel, les hématies sont moins nocives pour les amibes que le sérum.

Remarquons aussi que l'adjonction de sang total avec émétine a amené l'enkystement dans une culture qui n'en avait jamais présenté. Est-ce dû à l'action de la faible dose du médicament ? Ce serait un point intéressant à préciser à cause des doses faibles initiales employées en thérapeutique.

\section{RÉsumé}

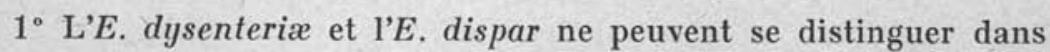
les cultures par leur différence de sensibilité à l'émétine. La toxicité de ce produit est la même pour les deux espèces in vitro.

$2^{\circ}$ Nous avons étudié l'action des divers médicaments sur les cultures d'amibes.

Les amibes sont tuées en $\mathbf{2 4}$ heures aux doses suivantes:

\begin{tabular}{|c|c|}
\hline Emetine & $500-1$ p. 1.000 \\
\hline Céphéline.. & 1 p. $50.000-1$ p. 75.000 \\
\hline Ipéca $\ldots \ldots \ldots \ldots \ldots$ & $50-1 \mathrm{p}$ \\
\hline $\begin{array}{l}\text { Iodure double d'émétine et } \\
\text { de bismuth } \ldots \ldots \ldots \ldots \ldots\end{array}$ & \\
\hline Stovarsol ................. & $600-1 \mathrm{p}$ \\
\hline Yatren................ & $200-1$ p. \\
\hline $914 \ldots \ldots \ldots \ldots \ldots \ldots$ & 1 p. $10.000-1$ p. 50.000 \\
\hline Iode............. & 1 p. .600 \\
\hline Sublimé... & $1 \mathrm{p}$. \\
\hline
\end{tabular}

$3^{\circ}$ Nous avons fait la critique de la méthode :

a) L'iode est beaucoup plus nocif pour les amibes in vitro, que l'expérimentation sur les cultures ne le prouve.

b) Le stovarsol semble plus actif en présence d'extrait de foie qu'ajouté directement aux cultures.

c) L'émétine a un pouvoir amibicide plus rapide en solution dans le sérum que dans une solution aqueuse ordinaire. 


\section{BIBLIOGRAPHIE}

Brumpt, - Etude sommaire de l'Entamœba dispar n. sp. Bull. Acad. Médecine. Paris, XCIV, 1925, p. 1.

- Individualité de l'Entamoba dispar. Bull. soc. path. exot., XIX, 1926, p. 399. Bовск et Drbohlav. - The cuItivation of Endamceba histolytica. Proc. Nat. Acad. Sc., II, 1925, p. 235.

- The cultivation of Endamaba histolylica. Amer Journ. of hygiene, V, 1925, p. 371 .

DoBell et Dale. - Experiments on the therapeutics of amœbic dysentery. Journ. Pharmacol. and exp. Therap., V, p. 339.

Dobell et Laidlaw, - On the cultivation of $E$. histolytica and some other Entozoic Amœba. Parasilology, XVIII, 1926, p. 283.

- The action of Ipecacuanha alkaloid on E. histolytica and some other Entozoic Amœbian culture. Parasitology, XVIII, 1926, p. 266.

Drвoнlav. - Une nouvelle preuve de la possibilité de cultiver $E$. dysenteriz type histolytica. Ann. Parasil., III, 1925, p. 349.

Kofoid et WAGENER. - Studies on the effect of certain drugs upon $E$. dysenterix in vitro. Univ. California Public Zool., XXVIII, 1923, p. 155.

Levaditi. - Le mécanisme d'action des dérivés arsenicaux dans les trypanosomiases. Bull. Institut Pasteur, XXIII, 1909, p. 604.

Maurel. - I. Action du chlorhydrate d'émétine sur les éléments figurés de notre sang. II. Note sur l'ordre de sensibilité et de toxicité des principaux éléments anatomiques sous l'influence de l'émétine. C. R. soc. biol., 1901, p. 996.

Rogers. - The rapid Cure of Amœbic dysentery and hepatitis by hypodermic injections of soluble salts of Emetine. Further experience of the specific curative action in Amœbic disease of hypodermic injections of soluble salt of Emetine. Brit Med. Journ., juin et août 1922, p. 1424.

Sellard et Leiva. - Experimental therapy of amœbic dysentery. Journ. Pharm. and experim. Therap. Baltimore, XXII, 1924, p. 467.

- Investigations concerning the treatment of amœbic dysentery. Philippine Journ. Sc., XXII, 1923, p. 37.

VEDDER. - An experimental study of the action of Ipecacuanha on Amœba. Far Easten Assoc. Trop. Med. Trans. Second Biennal Congress held at Hongkong 1912 , p. 87.

Vogel. - Ueber Kulturen der Ruhramöbe und deren Beeinflussung durch Yatren. Arch. f. Schiffs u. Trop. Hyg. XXXI, 1927, p. 74.

Yorke et Adass. - I. Developmont of cysts. Excistation and development of encysted amœba in vitro. II. Longevity of the cysts in vitro, and their resistance to heat and to various drugs and chemicals. An. Trop. Med. and Parasit., XX, 1926, p. 279.

Laboratoire de Parasitologie de la Facullé de médecine de Paris. 